\title{
MEDIDAS DE EFICIÊNCIA E RETORNO DE INVESTIMENTO: UM ESTUDO NAS DISTRIBUIDORAS DE ENERGIA ELETTRICA BRASILEIRAS COM BASE EM DATA ENVELOPMENT ANALYSIS, ÍNDICE DE MALMQUIST E ROI
}

\author{
MEASURES OF EFFICIENCY AND INVESTMENTS \\ RETURN: AN STUDY OF BRAZILIAN ELECTRICITY \\ DISTRIBUTORS WITH DATA ENVELOPMENT ANALYSIS, \\ MALMQUIST INDEX AND ROI
}

Recebido 10/02/2013 Aceito 10/04/2013

Valter Saurin ${ }^{1}$, Ana Lúcia Miranda Lopes ${ }^{2}$ Newton Carneiro Affonso da Costa Junior ${ }^{3}$, Carlos Alberto Gonçalves ${ }^{4}$

\section{RESUMO}

O presente texto tem como objetivo verificar, em um conjunto de empresas do setor elétrico brasileiro, se existe relação entre o retorno sobre o investimento (ROI) e o conceito de eficiência estimado pela metodologia Data Envelopment Analysis (DEA), bem como avaliar o crescimento de produtividade com base no Índice de Malmquist (Fare et al, 1996). A hipótese é a de que as empresas que obtiveram retorno sobre o investimento mais elevado durante determinado período de tempo são as que empregaram seus recursos de forma eficiente na obtenção de seus produtos (DEA eficientes). $O$ insumo (1) e os produtos (5) do modelo DEA correspondem ao custo operacional (I), à extensão da rede $(\mathrm{km})$, ao número de consumidores e ao mercado faturado de alta, média e baixa tensão, respectivamente. A amostra foi constituída por 31 empresas de distribuição de energia elétrica brasileiras e o período de realização do estudo foi de 2007 a 2009. Calculouse o índice de Malmquist (M0), representado pela mudança no fator de produtividade total (TFPC), o qual é composto pela evolução da eficiência técnica (EC) e pela mudança na fronteira tecnológica (TC) das empresas. Determinou-se também o ROI para cada período. Calculou-se a correlação entre as variáveis TFPC, TC, EC e Escore do DEA e o ROI no período de 2007 a 2009. Os resultados mostraram uma correlação positiva, porém fraca, entre variação da eficiência e os índices supracitados, negando a hipótese inicial.

Palavras-chave: Índice de Malmquist; Data Envelopment Analysis - DEA; ROI do setor elétrico.

${ }^{1}$ Graduado em Administração Pública pela Universidade Federal de Santa Maria - UFSM, graduado em Administração de Empresas pela Universidade Federal de Santa Maria - UFSM, graduado em Ciências Econômicas pela Faculdade de Ciências Politicas e Econômicas de Santa Maria, graduado em Ciências Contábeis pela Universidade Federal de Santa Maria - UFSM, mestre em Administração pela Universidade Federal do Rio Grande do Sul - UFRGS e doutor em Administração de Empresas pela Fundação Getúlio Vargas - FGV. Professor associado II da Universidade Federal de Santa Catarina UFSC. Florianópolis, Santa Catarina, Brasil. E-mail: inpeau@inpeau.ufsc.br. 2 Graduado em Engenharia Civil pela Pontifícia Universidade Católica - PUC/RS, possui mestrado e doutorado em Engenharia de Produção pela Universidade Federal de Santa Catarina - UFSC. Atualmente é professora Adjunto III do Departamento de Ciências Administrativas da Faculdade de Ciências Econômicas da Universidade Federal de Minas Gerais - FACE/UFMG e membro permanente dos cursos de mestrado e doutorado do Centro de Pós-Graduação e Pesquisas em Administração da Universidade Federal de Minas Gerais CEPEAD/UFMG. Belo Horizonte, Minas Gerais, Brasil. E-mail: analopes@face.ufmg.br.

${ }^{3}$ Graduado pela Escola Politécnica da Universidade de São Paulo - EPUSP, mestre em Engenharia de Produção pela Universidade Federal de Santa Catarina - UFSC e doutor em Administração pela Fundação Getúlio Vargas - FGV, com doutorado sandwich na Universidade de Lancaster, Reino Unido. Professor da Universidade Federal de Santa Catarina - UFSC. Florianópolis, Santa Catarina, Brasil. E-mail: newton@cse.ufsc.br.

${ }^{4}$ Graduado em Engenharia Civil pela Universidade Federal de Minas Gerais - UFMG, mestre em Informática pela Pontifícia Universidade Católica do Rio de Janeiro - PUC/RJ e doutor em Administração pela Universidade de São Paulo - USP. Professor Associado da Universidade Federal de Minas Gerais - UFMG e da Universidade FUMEC nas faculdades de Ciências Econômicas e Ciências Empresariais. Belo Horizonte, Minas Gerais, Brasil: E-mail: carlos@face.ufmg.br. 


\section{ABSTRACT}

The present study was to examine, from two case studies, management processes (strategic planning, management control systems and human resources) and cultural (succession and organizational culture) two family businesses in the state of São Paulo. Concepts were discussed, and specific features, as well as factors related to organizational management of family businesses (planning, control, people management, culture and succession) to support the studies described. Through interviews and observation of routine business, it was concluded that there is evidence that the management in Company " $B$ " moving towards professionalization, due to be developing a formal strategic planning. This company already has clear goals for employees and creating an area of people management. In contrast, Company " $A$ ", still needs to improve its organizational structure, as there is lack of planning, strategies and formal actions, so that decisions are focused on owners and taken in light of action-reaction.

Keywords: Family businesses; Succession process; Organizational culture; Strategic planning; Human resource management;

\section{INTRODUÇÃO}

Um dos mais importantes princípios de qualquer negócio é o princípio da eficiência, em que os melhores possíveis efeitos econômicos (produtos) são alcançados com os menores possíveis sacrifícios (insumos) (MARTIC et al., 2009). Avaliar a eficiência de setores sob regulação, como o de energia, vem chamando a atenção de pesquisadores e governos. No caso brasileiro, tem-se observado mudanças significativas desde a privatização ocorrida durante os anos de 1990 e a criação da Agência Nacional de Energia Elétrica (ANEEL) em 1997. Dentre as metas que orientam o trabalho da ANEEL, está o de incentivar a eficiência das empresas de energia elétrica a fim de garantir o fornecimento de energia de qualidade a um baixo custo ao povo brasileiro.

Seguindo a tendência mundial de passagem de modelos de empresa de referência para modelos de benchmarking, a ANEEL propôs, recentemente, uma metodologia para o cálculo dos custos operacionais regulatórios com base na análise de fronteira (NT 265/2010). A metodologia Data Envelopment Analysis (DEA) foi, então, proposta para o cálculo da eficiência das empresas de distribuição de energia, assim como para a mensuração da mudança de produtividade através do índice de Malmquist (Caves, Christensen e Diewert, 1982). As duas medidas fazem parte do Fator X a ser utilizado no 3o ciclo de revisão tarifária periódica (3CRTP).

Vários trabalhos apontam a adequabilidade da metodologia DEA na análise de eficiência de empresas do setor de energia. Entre eles, podem-se citar: Wang et al. (2007), que analisam a eficiência e o desempenho de empresas de energia elétrica e seus efeitos nos preços ao consumidor; Delmas e Tokat (2002), que verificam os efeitos da desregulamentação no setor de distribuição de energia elétrica; Sarkis et al. (2009), que determinam a eficiência relativa do ponto de vista técnico e ambiental de 437 empresas geradoras de energia nos Estados Unidos; Cherchye et al (2003), que analisam os avanços metodológicos do DEA, bem como os pontos fortes e fracos, ilustrando tal análise com o caso de aplicação do DEA na determinação das tarifas do setor elétrico na Holanda; Zhang et al. (1998), que investigam a eficiência da distribuição de energia elétrica na Austrália, Suécia e Nova Zelândia; e Resende (2002), que mensura a eficiência de distribuidoras de energia elétrica brasileiras em 1997 e 1998. Uma síntese de pesquisas de DEA em energia e em estudos ambientais pode ser encontrada em Zhou, Ang e Poh (2008).

Ressalta-se ainda o estudo de Ariff et al. (2009), que aplicam a DEA para testar o desempenho de uma abrangente amostra de empresas de telecomunicações de diversos países, quatro anos antes e quatro anos depois de terem sido privatizadas. Neste estudo foram 
identificadas melhorias significantes na eficiência financeira e operacional após a privatização.

Com referência ao uso de DEA em contabilidade, tem-se o estudo de Santos e Casa Nova (2005), que apresentam um modelo partindo de informações contidas em demonstrações contábeis de empresas do setor elétrico brasileiro, coletadas da base de dados Melhores e Maiores da Fipecafi-Exame para os anos de 1999 e 2000. Os escores de eficiência obtidos pela aplicação do modelo foram comparados com o indicador de Excelência Empresarial de Melhores e Maiores e com o Retorno sobre o Patrimônio Líquido, indicador contábil tradicional, explorando as vantagens e limitações do uso do DEA.

Por último, cita-se o estudo conduzido por Zhu (2000) acerca do desempenho financeiro das empresas constantes na Fortune 500 com o emprego do DEA. Os dados foram coletados da edição da Revista Fortune, de abril de 1996, e os resultados apontaram para o fato de que somente $3 \%$ das empresas que são do nível mais alto com base na receita estão operando com o desempenho otimizado.

Nesse contexto, o presente estudo tem como objetivo verificar, em um conjunto de empresas do setor elétrico brasileiro, se existe relação entre criação de valor, medido pelo retorno sobre o investimento (ROI), e o conceito de eficiência, estimado pela metodologia DEA. Espera-se, ainda, avaliar o crescimento de produtividade com base no Índice de Malmquist (Fare et al., 1994) durante o período de 2007 a 2009.

Este estudo, além desta introdução, contém uma seção destinada à metodologia DEA e uma seção destinada às formulações e aos conceitos de mudança de produtividade. Contém, também, um sumário sobre o ROI, assim como a metodologia empregada para a execução deste trabalho. Posteriormente, traz uma análise e interpretação dos resultados e, finalmente, as considerações finais.

\section{REVISÃO DE LITERATURA}

\subsection{Análise da Eficiência Relativa por meio de Data Envelopment Analysis (DEA)}

O problema de quanto uma empresa pode esperar aumentar seus produtos (resultados) simplesmente aumentando sua eficiência, sem que, para isto, tenha de consumir maiores quantidades de insumos (recursos), foi abordado por Farrel (1957). O propósito de seu trabalho era fornecer uma medida de eficiência produtiva satisfatória que levasse em conta todos os insumos de uma empresa, evitando problemas de cálculo com índices, e mostrar como isto poderia ser computado na prática por meio de uma função de produção.

Generalizando a medida de eficiência técnica de Farrell (1957), para casos de múltiplos produtos e múltiplos insumos, Charnes, Cooper e Rhodes (1978) propõem a metodologia Data Envelopment Analysis (DEA). Esta metodologia foi aplicada inicialmente em instituições públicas em que a dificuldade de alocação de preços a insumos e produtos impossibilitava a mensuração de sua eficiência. Mais recentemente, sua aplicação estendeu-se a empresas privadas e áreas como aviação, agricultura, energia, setor bancário, entre outras.

DEA é uma ferramenta baseada em programação linear que mede a eficiência relativa de unidades tomadoras de decisão (DMU's). O pressuposto de DEA é que estas unidades são comparáveis. Além da identificação da eficiência relativa, DEA identifica as fontes e os níveis de ineficiência para cada um dos insumos e produtos observados (MARTIC et al., 2009). De maneira objetiva, pode-se dizer que DEA determina as melhores condições de operação para cada unidade produtiva separadamente, de modo a maximizar a sua eficiência, aplicando as mesmas condições 
às demais unidades do grupo em análise. Aquelas unidades produtivas para as quais o escore de eficiência relativa, calculado a partir de pesos obtidos pelo próprio modelo de programação linear, é igual ou mais favorável que os índices calculados para as outras unidades em análise são consideradas eficientes.

Resolvendo-se o problema, sucessivamente, para todas as unidades produtivas, obtémse um subconjunto das unidades consideradas eficientes, que servirá de base para a determinação da fronteira de eficiência e para o estabelecimento de metas para as unidades ineficientes. Compara-se, portanto, cada unidade apenas com as semelhantes de melhor desempenho, ou seja, aquelas situadas na fronteira de eficiência. Qualquer unidade produtiva incluída ou excluída do conjunto em análise modifica o conjunto de produção e, em decorrência, pode modificar a fronteira. As unidades que servem de referenciais para estabelecimento de metas, ou comparação direta, são denominadas benchmarks ou peer's.

Charnes, Cooper e Rhodes (1978) propuseram um modelo que assumia retornos constantes de escala, denominado CRS (Constant Returns to Scale) ou CCR (em homenagem aos autores). Textos posteriores apresentaram conjuntos diferentes de suposições, dos quais se destaca o modelo desenvolvido por Banker, Charnes e Cooper (1984), que assumia retorno variável de escala, denominado VRS (Variable Returns to Scale) ou BCC. No que diz respeito à orientação, ambos os modelos podem ser classificados como orientados a insumos ou a produtos, conforme ocorre a busca das metas pelas unidades ineficientes.

O modelo representado abaixo (1) é conhecido como modelo CCR (Charnes, Cooper e Rhodes, 1978) dos multiplicadores. Em DEA e, consequentemente, neste modelo, $y_{\mathrm{rj}}$ e $x_{\mathrm{ij}}$ representam, respectivamente, o r-ésimo produto e o i-ésimo insumo da j-ésima unidade tomadora de decisão (DMU). Os $u_{r}$ e $v_{i}$ são os pesos do insumo $i$ e produto $r$ (coeficientes de ponderação) determinados pela solução do problema. Este modelo busca otimizar a relação insumo/produto da unidade $k$, atribuindo livremente pesos aos insumos e aos produtos, tendo como restrição o fato de que, com os mesmos pesos, todas as DMU's (inclusive a unidade $k$ ) não apresentem esta relação menor que 1 (TORESAN, 1998).

$$
\begin{aligned}
& \text { Min } E F_{k}=\sum_{i=1}^{m} v_{i} x_{i k} \\
& \text { Sujeito a: } \\
& \sum_{r=1}^{s} u_{r} y_{r k}=1 \\
& -\sum_{r=1}^{s} u_{r} y_{r j}+\sum_{i=1}^{m} \mathrm{v}_{\mathrm{i}} x_{i j} \geq 0, j=1, \ldots, n \\
& u_{r}, v_{i \geq 0}, \quad \text { para } r=1, \ldots, n \text { e } i=1, \ldots, m
\end{aligned}
$$

Neste modelo, os pesos transformam os produtos e insumos em medidas únicas chamadas "produto virtual" e "insumo virtual". A medida de desempenho de cada DMU é otimizada através de $n$ resoluções do modelo de programação linear (uma para cada $\mathrm{DMU}_{\mathrm{j}}$ ), gerando uma medida de eficiência para cada unidade em análise. Desse modo, uma unidade $k$ será considerada eficiente sempre que $E F_{k}=1$. 
Já o modelo BCC de Banker, Charnes e Cooper (1984) ou VRS pressupõe que as unidades avaliadas apresentam retornos variáveis de escala. Este modelo, na forma dual do modelo dos multiplicadores chamada envelopamento, pode ser representado da seguinte maneira (2):

$$
\begin{aligned}
& E f^{*}=\max E f \\
& \text { restrições : } \\
& \sum_{j=1}^{n} \lambda_{j} x_{i j} \leq x_{i o}, \quad i=1,2, \ldots, k \\
& \sum_{j=1}^{n} \lambda_{j} y_{r j} \geq \text { Ef } y_{\text {ro }}, \quad r=1,2, \ldots, m \\
& \sum_{j=1}^{n} \lambda_{j}=1, \quad \lambda_{j} \geq 0
\end{aligned}
$$

Neste modelo, o valor de $E f$ indica o grau de expansão radial possível para todos os produtos, dado o nível observado de insumos, caracterizando-se, portanto, como um indicador de eficiência relativa. Os vetores de insumos $i$ e produtos $r$ da unidade $j$ são representados por $x_{i j}$ e $y_{r j}$ e as unidades de referência que compõem a faceta de projeção são designadas por $\lambda$. Se $E f=1$, então a unidade está sobre a fronteira de eficiência, podendo servir de referência para as demais. Por outro lado, se $E f>1$, então a unidade $j$ pode ainda aumentar seus produtos, mantendo inalterados os insumos e, portanto, sendo ineficiente perante o grupo de unidades analisado.

Em outras palavras, cada unidade é comparada com uma unidade virtual obtida por combinação linear de todas as unidades do grupo. Assim, cada insumo e produto desta unidade virtual são a combinação linear dos insumos e produtos de todas as unidades do grupo. $O$ valor de $E f$, neste caso, é sempre maior ou igual a um. A existência de um valor de $E f$ maior que um indica a possibilidade de construção de uma unidade virtual que produz mais, utilizando igual (ou menor) quantidade de insumos que a unidade analisada. Em outras palavras, em um modelo orientado produto, procura-se expandir radialmente o vetor de produtos, tanto quanto possível, para cada unidade em análise. O limite é a fronteira de eficiência estimada para o conjunto de pontos observados, os quais são determinados pelas outras unidades produtivas. Este problema deve ser resolvido para cada unidade, gerando seu escore de eficiência relativa.

A última restrição deste modelo ( $\left.\sum_{j=1}^{N} \lambda_{j}=1\right)$ faz dele uma envoltória VRS.

Sem esta restrição, o modelo considera uma envoltória CRS.Em relação à orientação a insumos ou produtos, é importante observar que, se uma unidade for eficiente no modelo orientado a produto, também o será no modelo CRS orientado a insumo. Entretanto, dependendo da orientação utilizada, as unidades de referência e os indicadores de retração de insumos ou expansão de produtos, para as unidades ineficientes, podem ser diferentes (BHAT ET AL., 2001).

\section{2 Índice de Malmquist $\left(M_{0}\right)$}

Neste estudo, usa-se o índice de Malmquist $\left(\mathrm{M}_{0}\right)$ para medir a mudança de produtividade das empresas distribuidoras de energia elétrica brasileiras. O conceito deste índice foi apresentado por Malmquist em 1953, tendo sido estudado e desenvolvido desde então em modelos não paramétricos por diversos autores, dos quais se destacam, entre outros, Caves et al. (1982), 
Färe et al. $(1989,1992$, , 1994, 1986b, 2000). O M representa a mudança na produtividade total dos fatores (Total Factor Productivity Change - TFPC) de uma DMU. Reflete o progresso ou a redução na eficiência técnica (1) e o progresso ou a redução da fronteira tecnológica (2) entre dois períodos de tempo em uma estrutura de múltiplos insumos e produtos. Este índice é composto pela mudança na eficiência (Efficiency Change - EC) e pela mudança na fronteira tecnológica (Technological Change - TC) da DMU.

A EC da DMU considera o aumento da eficiência técnica produtiva ao longo do tempo, correspondendo ao resultado de melhorias contínuas nos processos de produção e nos produtos, utilizando-se de uma mesma tecnologia. Já a TC é resultante da introdução de tecnologias que produzem um produto melhor com menor utilização de insumos. O fator de produtividade total (TFPC) é a média geométrica dos dois índices - EC e TC.

O TFPC parte da estimativa da chamada Função Distância de Shephard, que, em sua versão orientada para produto e considerando o período $t$ como referência, pode ser descrita de acordo com a equação (3):

$$
D_{0}^{t}\left(x^{t}, y^{t}\right)=\inf \left\{\theta:\left(x^{t}, y^{t}\right) / \theta \in S^{t}\right\}
$$

Onde $x$ é quantidade de insumo, $y$ é a quantidade de produto e $S$ é o conjunto de possibilidade de produção.

Caves, Christensen e Diewert (1982) propõem medir os ganhos de produtividade ao longo do tempo utilizando as equações (4) e (5):

$$
\begin{aligned}
& M^{t-1}=\frac{D_{0}^{t-1}\left(x^{t}, y^{t}\right)}{D_{0}^{t-1}\left(x^{t-1}, y^{t-1}\right)} \\
& M^{t}=\frac{D_{0}^{t}\left(x^{t}, y^{t}\right)}{D_{0}^{t}\left(x^{t-1}, y^{t-1}\right)}
\end{aligned}
$$

O índice de Malmquist de TFPC será, então, a média geométrica de (4) e (5), conforme a equação (6):

$$
M_{0}\left(x^{t+1}, y^{t+1}, x^{t}, y^{t}\right)=\left(\frac{D_{0}^{t-1}\left(x^{t}, y^{t}\right)}{D_{0}^{t-1}\left(x^{t-1}, y^{t-1}\right)} \cdot \frac{D_{0}^{t}\left(x^{t}, y^{t}\right)}{D_{0}^{t}\left(x^{t-1}, y^{t-1}\right)}\right)^{\frac{1}{2}}
$$

Nesta equação, $M_{0}$ pode assumir valores que representam queda de produtividade $\left(M_{0}<1\right)$, ausência de mudança de produtividade $\left(M_{0}=1\right)$ e crescimento de produtividade $\left(M_{0}>1\right)$.

Fare, Grosskopf, Lindgren e Roos (1994) decompõem o índice de Malmquist em suas componentes de eficiência técnica (EC) e mudança tecnológica (TC), originando o primeiro e o segundo termo da equação (7):

$$
M_{0}\left(x^{t+1}, y^{t+1}, x^{t}, y^{t}\right)=\frac{D_{0}^{t+1}\left(x^{t+1}, y^{t+1}\right)}{D_{0}^{t}\left(x^{t}, y^{t}\right)} \cdot\left[\frac{D_{0}^{t}\left(x^{t+1}, y^{t+1}\right)}{D_{0}^{t+1}\left(x^{t+1}, y^{t+1}\right)} \cdot \frac{D_{0}^{t}\left(x^{t}, y^{t}\right)}{D_{0}^{t+1}\left(x^{t}, y^{t}\right)}\right]^{1 / 2}
$$


Ainda em 1994, Fare et al. relaxaram a hipótese de retornos constantes a escala, permitindo retornos variáveis (VRS) e introduzindo, então, o conceito de DEA no cálculo da mudança de produtividade, conforme a equação (8), transcrita abaixo:

$M_{0}\left(x^{t+1}, y^{t+1}, x^{t}, y^{t}\right)=\left(\frac{D_{0 V}^{t+1}\left(x^{t+1}, y^{t+1}\right)}{D_{0 V}^{t}\left(x^{t}, y^{t}\right)}\right) \cdot \frac{\left(\frac{D_{0}^{t+1}\left(x^{t+1}, y^{t+1}\right)}{D_{0 V}^{t+1}\left(x^{t+1}, y^{t+1}\right)}\right)}{\left(\frac{D_{0}^{t}\left(x^{t}, y^{t}\right)}{D_{0 V}^{t}\left(x^{t}, y^{t}\right)_{0 V}}\right)}\left[\frac{D_{0}^{t}\left(x^{t+1}, y^{t+1}\right)}{D_{0}^{t+1}\left(x^{t+1}, y^{t+1}\right)} \cdot \frac{D_{0}^{t}\left(x^{t}, y^{t}\right)}{D_{0}^{t+1}\left(x^{t}, y^{t}\right)}\right]^{1 / 2}$

Segundo a ANEEL (NT 267/2010), o subscrito "V" na equação anterior, assim como os demais subscritos, significa VRS.

\subsection{Retorno sobre o investimento (ROI)}

A formulação básica do retorno sobre o investimento (ROI) consiste no lucro líquido operacional (EBIT) em relação ao investimento total. O ROI representa o retorno sobre o capital próprio e de terceiros aplicados no empreendimento.

O investimento total compreende o valor do capital próprio mais o valor da dívida total, isto é, o capital de terceiros. Nas empresas de capital aberto, emprega-se o valor de mercado do capital próprio e, nas empresas de capital fechado, usa-se o valor do patrimônio líquido. 0 capital de terceiros é representado pela dívida total. Considera-se que o valor contábil do exigível oneroso, resultante de financiamentos, representa o capital de terceiros.

Nesta formulação, o ROI é uma medida de rentabilidade que permite estabelecer a classificação das empresas e correlacioná-las com o escore obtido pelo DEA. Considerando que o ROI é um índice percentual, resultante do lucro líquido operacional em relação ao investimento total de encerramento do exercício fiscal da empresa, não há necessidade de fazer qualquer correção monetária dos respectivos valores. Portanto, a formulação empregada é dada pela equação (9):

$$
R O I=\frac{E B I T}{\text { Investimento }}
$$

Onde ROI é retorno sobre o investimento total, EBIT é o lucro líquido operacional e Investimento é o capital próprio mais o capital de terceiros.

\section{METODOLOGIA}

A amostra deste estudo compreende as empresas de distribuição de energia elétrica, de capital aberto e fechado, que apresentaram todos os dados necessários para o cálculo de sua eficiência. Nestes critérios, incluem-se 31 empresas com atividade de distribuição de energia elétrica.

Os dados básicos para os cálculos do escore de eficiência relativa determinada pela Data Envelopment Analysis (DEA) e pelo retorno sobre o investimento (ROI) são referentes ao encerramento do exercício fiscal de cada ano no período de 2007 a 2009 e foram coletados no banco de dados da Economática e da Comissão de Valores Mobiliários (CVM). Estes dados encontram-se nos Anexos I, II e II.

A escolha das variáveis (insumos e produtos) para a determinação da eficiência relativa (DEA) das empresas foi baseada na opinião de especialistas que consideraram a relevância das variáveis que refletem os recursos colocados e os resultados das respectivas empresas. 
A literatura sobre DEA não tem se aprofundado no problema da seleção de variáveis e a grande maioria dos trabalhos publicados aborda esse tema com base na opinião de especialistas ou até mesmo na disponibilidade de dados, segundo Angulo-Meza et al. (2007). Uma revisão de métodos de seleção de variáveis pode ser vista em Senra (2004), Senra et al. (2007) e AnguloMeza et al. (2007). No setor elétrico, Wang et al. (2007) empregaram, como insumos, o montante de investimentos $(\$ M)$ e o número de trabalhadores e, como produtos, as vendas de energia ( $\mathrm{kWh}$ ) e a densidade dos consumidores (consumidores por km2).

No presente estudo, o insumo corresponde ao custo operacional, que inclui os gastos com pessoal e despesas de manutenção. Este insumo está coerente com a análise do DEA, que considera eficiente a unidade que alcança os maiores valores para os produtos com uma utilização mínima dos recursos necessários.

Os produtos incluem a extensão da rede em km, o número de consumidores (quantidade), o valor faturado do mercado de alta tensão, o mercado de média tensão e o mercado de baixa tensão. O modelo DEA empregado para determinar o nível de eficiência das empresas de energia elétrica é o VRS orientado à minimização de insumos. A orientação insumo é adotada por ser a mais adequada para análise de empresas de energia elétrica que atuam em setor regulamentado. Estas empresas, em geral, têm uma taxa de retorno máxima preestabelecida. Assim, a melhor estratégia a ser adotada seria a otimização de seus custos/insumos. A formulação do ROI na forma percentual é empregada para determinar o retorno, uma vez que quanto maior o ROI melhor é o retorno do capital utilizado para alcançar este nível de resultados.

A análise pretendida neste estudo consiste em verificar a correlação entre os índices de eficiência estimados pelo DEA (média do escore de eficiência), o fator de produtividade total, dividido em seus três componentes, e a média do ROI no período de 2007 a 2009.

\section{RESULTADOS}

$\mathrm{Na}$ primeira parte deste item, apresentam-se os resultados do escore da Data Envelopment Analysis (DEA), do Índice de Malmquist $\left(\mathrm{M}_{0}\right)$ e do retorno sobre o investimento (ROI) das empresas analisadas. Em seguida, verifica-se a correlação entre os índices e o ROI no período de 2007 a 2009.

\subsection{Análise dos resultados}

A Tabela I demonstra os resultados dos escores de eficiência técnica com base na DEA, no período de 2007 a 2009, e a média destes três anos para cada empresa. Uma empresa que tenha obtido um escore igual 1 (um) é considerada eficiente no modelo DEA.

Tabela 1 - Escore de eficiência - período 2007 a 2009

\begin{tabular}{|l|c|c|c|c|}
\hline \multicolumn{1}{|c|}{ Empresa } & $\mathbf{2 0 0 7}$ & $\mathbf{2 0 0 8}$ & $\mathbf{2 0 0 9}$ & Média \\
\hline E01 AES SUL & 1 & 0,9176 & 0,8760 & 0,9312 \\
\hline E02 AMAZONAS & 0,6300 & 0,5699 & 0,5320 & 0,5773 \\
\hline E03 AMPLA & 0,5944 & 0,6059 & 0,6122 & 0,6042 \\
\hline E04 BANDEIRANTE & 0,8901 & 0,8074 & 0,7381 & 0,8119 \\
\hline E05 CEAL & 0,5965 & 0,5789 & 0,4802 & 0,5519 \\
\hline E06 CEB & 0,6698 & 0,7308 & 0,6902 & 0,6969 \\
\hline E07 CEEE & 0,6362 & 0,6171 & 0,5229 & 0,5921 \\
\hline
\end{tabular}




\begin{tabular}{|l|c|c|c|c|}
\hline \multicolumn{1}{|c|}{ Empresa } & $\mathbf{2 0 0 7}$ & $\mathbf{2 0 0 8}$ & $\mathbf{2 0 0 9}$ & Média \\
\hline E08 CELESC & 0,7294 & 0,7212 & 0,8373 & 0,7626 \\
\hline E09 CELG & 0,6345 & 0,6256 & 0,5867 & 0,6156 \\
\hline E10 CELPA & 0,5281 & 0,5701 & 0,4655 & 0,5212 \\
\hline E11 CELPE & 0,8153 & 0,8466 & 0,8547 & 0,8389 \\
\hline E12 CELTINS & 0,9562 & 1 & 1 & 0,9854 \\
\hline E13 CEMAR & 1 & 0,9926 & 0,9358 & 0,9761 \\
\hline E14 CEMAT & 0,7624 & 0,7908 & 0,7547 & 0,7693 \\
\hline E15 CEMIG & 1 & 1 & 1 & 1 \\
\hline E16 CEPISA & 0,6315 & 0,6339 & 0,5742 & 0,6132 \\
\hline E17 COELBA & 1 & 1 & 1 & 1 \\
\hline E18 COELCE & 0,8508 & 0,8030 & 0,8473 & 0,8337 \\
\hline E19 COPEL & 1 & 0,9780 & 0,9502 & 0,9761 \\
\hline E20 COSERN & 0,9755 & 1 & 1 & 0,9918 \\
\hline E21 CPFL PAULISTA & 1 & 1 & 1 & 1 \\
\hline E22 ELEKTRO & 0,819 & 0,7479 & 0,7443 & 0,7704 \\
\hline E23 ELETROPAULO & 1 & 1 & 1 & 1 \\
\hline E24 EMG & 1 & 1 & 1 & 1 \\
\hline E25 ENERSUL & 0,6603 & 0,7706 & 0,7622 & 0,7310 \\
\hline E26 EPB & 0,9077 & 0,8825 & 0,8557 & 0,8820 \\
\hline E27 ESCELSA & 0,9679 & 0,9845 & 0,8904 & 0,9476 \\
\hline E28 ESSE & 0,9919 & 0,8659 & 0,9083 & 0,9220 \\
\hline E29 LIGHT & 0,8743 & 0,9839 & 0,9794 & 0,9459 \\
\hline E30 PIRATININGA & 1 & 1 & 1 & 1 \\
\hline E31 RGE & 1 & 1 & 1 & 1 \\
\hline
\end{tabular}

Fonte: Resultados do cálculo dos escores de eficiência.

Nesta tabela, observa-se que, de um total de 31 , somente 10 empresas (30\%) atingiram a média de $100 \%$ de eficiência e apenas sete tiveram o índice constante no período analisado: CEMIG, COELBA, CPFL PAULISTA, ELETROPAULO, EMG, PIRATININGA e RGE. Ressalta-se que a CELTINS e a COSERN foram eficientes na média e nos períodos de 2008 e 2009.

A Tabela 2 demonstra os resultados do índice de Malmquist $\left(\mathrm{M}_{0}\right)$ no período de 2007 a 2009, período em que, das 31 empresas, 19 (61\%) obtiveram a evolução do fator de produtividade total (Total Factor Productivity Change - TFPC) igual ou acima de um. A empresa que teve este índice igual um permaneceu constante, maior do que um aumentou sua produtividade e menor do que um reduziu sua posição quanto à eficiência total. Este resultado é composto pelo produto dos respectivos índices de evolução da eficiência técnica (Efficiency Change - EC) e de mudança da fronteira tecnológica (Technological Change - TC).

Nesta tabela verifica-se, também, que, enquanto 14 empresas (45\%) apresentaram o índice EC igual ou maior do que um,, todas as empresas tiveram o índice TC igual ou maior do que um. Estes indicadores permitem identificar o ponto forte ou fraco da empresa em relação às demais, uma vez que o resultado é a eficiência relativa e não absoluta em cada período de 
MEDIDAS DE EFICIÊNCIA E RETORNO DE INVESTIMENTO: UM ESTUDO NAS

DISTRIBUIDORAS DE ENERGIA ELÉTRICA BRASILEIRAS COM BASE EM

DATA ENVELOPMENT ANALYSIS, ÍNDICE DE MALMQUIST E ROI

Tabela 2 - Índice de Malquist $\left(\mathrm{M}_{0}\right)$ - período de 2007/2009 estudo.

\begin{tabular}{|c|c|c|c|}
\hline Empresa & TC & EC & TFPC \\
\hline E01 AES SUL & 1,06 & 0,89 & 0,94 \\
\hline E02 AMAZONAS & 1,13 & 0,81 & 0,91 \\
\hline E03 AMPLA & 1,13 & 1,02 & 1,16 \\
\hline E04 BANDEIRANTE & 1,09 & 0,92 & 1 \\
\hline E05 CEAL & 1,2 & 0,74 & 0,89 \\
\hline E06 CEB & 1,1 & 1,12 & 1,23 \\
\hline E07 CEEE & 1,09 & 0,84 & 0,91 \\
\hline E08 CELESC & 1,06 & 1,04 & 1,09 \\
\hline E09 CELG & 1,06 & 1,08 & 1,15 \\
\hline E10 CELPA & 1,13 & 0,85 & 0,97 \\
\hline E11 CELPE & 1,19 & 1,04 & 1,23 \\
\hline E12 CELTINS & 1,02 & 1,13 & 1,15 \\
\hline E13 CEMAR & 1,09 & 0,88 & 0,96 \\
\hline E14 CEMAT & 1,06 & 1,17 & 1,24 \\
\hline E15 CEMIG & 1,06 & 0,89 & 0,94 \\
\hline E16 CEPISA & 1,06 & 0,88 & 0,93 \\
\hline E17 COELBA & 1 & 1 & 1 \\
\hline E18 COELCE & 1,21 & 0,96 & 1,15 \\
\hline E19 COPEL & 1,07 & 0,85 & 0,9 \\
\hline E20 COSERN & 1,14 & 1,04 & 1,18 \\
\hline E21 CPFL PAULISTA & 1,02 & 1 & 1,02 \\
\hline E22 ELEKTRO & 1,08 & 1 & 1,08 \\
\hline E23 ELETROPAULO & 1,07 & 0,92 & 0,98 \\
\hline E24 EMG & 1,08 & 0,99 & 1,07 \\
\hline E25 ENERSUL & 1,05 & 1,15 & 1,21 \\
\hline E26 EPB & 1,11 & 0,87 & 0,97 \\
\hline E27 ESCELSA & 1,05 & 0,93 & 0,97 \\
\hline E28 ESSE & 1,14 & 0,88 & 1 \\
\hline E29 LIGHT & 1,08 & 1,22 & 1,32 \\
\hline E30 PIRATININGA & 1 & 1 & 1 \\
\hline E31 RGE & 1 & 1 & 1 \\
\hline
\end{tabular}

Legenda: $\mathrm{TC}=$ mudança tecnológica; $\mathrm{EC}=$ mudança na eficiência; TFPC = produtividade total dos fatores

Como exemplo, verifica-se que a empresa LIGHT se mostrou eficiente em todos os anos analisados (Tabela 2), obteve um TFPG igual a 1,32, EC igual a 1,22 e TC igual a 1,08. Este resultado indica que a empresa apresentou resultado positivo em relação à evolução da eficiência técnica e à mudança da fronteira tecnológica. O fator de produtividade total (TFPC) é o resultado da evolução da eficiência técnica (EC) e da evolução da fronteira tecnológica (TC) da respectiva DMU. Outro exemplo é o da empresa CEMIG, que obteve índice de 0,89 na EC e resultado positivo na TC, com índice de 1,06, resultando em um TFPC de 0,94.

A Tabela 3 demonstra o retorno sobre o investimento (ROI), no período de 2007 a 2009, e a respectiva média. $O$ retorno foi obtido pelo lucro operacional (EBIT) em relação ao investimento total. Observa-se, nesta tabela, que, de 31 empresas, 28 (90\%) obtiveram um ROI médio positivo e apenas três tiveram um ROI negativo, destacando-se a PIRATININGA, que obteve um retorno médio excepcional de 105,20\%. 
Tabela 3 - Retorno sobre o investimento (ROI)

\begin{tabular}{|c|c|c|c|c|}
\hline Empresas & 2007 & 2008 & 2009 & Média \\
\hline E01 AES SUL & 0,2207 & 0,2367 & 0,2288 & 0,2287 \\
\hline E02 AMAZONAS & $-0,8405$ & 0,3117 & 0,0244 & $-0,1681$ \\
\hline E03 AMPLA & 0,2361 & 0,2915 & 0,2838 & 0,2704 \\
\hline E04 BANDEIRANTE & 0,3689 & 0,3542 & 0,3390 & 0,3540 \\
\hline E05 CEAL & 0,1415 & 0,1480 & 0,0228 & 0,1041 \\
\hline E06 CEB & 2,6747 & 0,8881 & 0,1628 & 1,2419 \\
\hline E07 CEEE & 0,0338 & $-0,0707$ & $-0,0879$ & $-0,0416$ \\
\hline E08 CELESC & 0,2545 & 0,1551 & 0,0482 & 0,1526 \\
\hline E09 CELG & $-0,0131$ & 0,0005 & $-0,1604$ & $-0,0577$ \\
\hline E10 CELPA & 0,1504 & 0,0748 & 0,1047 & 0,1099 \\
\hline E11 CELPE & 0,3535 & 0,3975 & 0,3539 & 0,3683 \\
\hline E12 CELTINS & 0,0752 & 0,1075 & 0,0772 & 0,0866 \\
\hline E13 CEMAR & 0,5908 & 0,4752 & 0,4075 & 0,4912 \\
\hline E14 CEMAT & 0,1839 & 0,1753 & 0,1561 & 0,1718 \\
\hline E15 CEMIG & 0,4859 & 0,4368 & 0,1858 & 0,3695 \\
\hline E16 CEPISA & $-0,0440$ & $-0,1130$ & 0,1636 & 0,0022 \\
\hline E17 COELBA & 0,5893 & 0,5512 & 0,4776 & 0,5394 \\
\hline E18 COELCE & 0,3103 & 0,3263 & 0,3400 & 0,3255 \\
\hline E19 COPEL & 0,2593 & 0,1991 & 0,0779 & 0,1788 \\
\hline E20 COSERN & 0,4634 & 0,3768 & 0,3213 & 0,3872 \\
\hline E21 CPFL PAULISTA & 1,5997 & 0,9548 & 0,9288 & 1,1611 \\
\hline E22 ELEKTRO & 0,6109 & 0,4592 & 0,4289 & 0,4997 \\
\hline E23 ELETROPAULO & 0,3341 & 0,3578 & 0,2987 & 0,3302 \\
\hline E24 EMG & 0,0689 & 0,0918 & 0,1182 & 0,0930 \\
\hline E25 ENERSUL & $-0,0857$ & 0,1842 & 0,2450 & 0,1145 \\
\hline E26 EPB & 0,1849 & 0,0988 & 0,1600 & 0,1479 \\
\hline E27 ESCELSA & 0,2268 & 0,2416 & 0,2419 & 0,2368 \\
\hline E28 ESSE & 0,1833 & 0,0543 & $-0,0641$ & 0,0578 \\
\hline E29 LIGHT & 0,2477 & 0,3662 & 0,2559 & 0,2899 \\
\hline E30 PIRATININGA & 1,6645 & 0,9716 & 0,5200 & 1,0520 \\
\hline E31 RGE & 0,1788 & 0,1879 & 0,2186 & 0,1951 \\
\hline
\end{tabular}

Fonte: elaborado pelos autores.

4.2 Análise de correlações

Considerando que os índices apresentam distribuições de probabilidade diferentes da normal, foi estimada a correlação de postos de Spearman, que é uma medida não paramétrica do ROI com a TC, a EC, o TFPC e o Escore do DEA. Para verificar se existe relação entre as respectivas variáveis, determinaram-se os índices de correlação, que se encontram na Tabela 4.

Nesta Tabela, constam os resultados das correlações entre o ROI médio com a evolução (ou não) na EC; com a evolução (ou não) da TC; com a mudança no TFPC; e com Escore de eficiência com base no DEA médio, no período de 2007 a 2009 de cada DMU. Verifica-se que a correlação 
entre o ROI médio e o escore de eficiência com base no DEA e entre o ROI médio e o índice EC foi significativa, obtendo, respectivamente, $5 \%$ e $10 \%$ de significância. As demais correlações não foram significativas.

Tabela 4 - Correlações (Spearman) 2007-2009

\begin{tabular}{c|cccc}
\hline & TC & EC & TFPG & $\begin{array}{c}\text { Escore } \\
\text { DEA }\end{array}$ \\
\hline \multirow{2}{*}{ ROI } & $-0,12$ & $0,31^{*}$ & 0,29 & $0,50 * * *$ \\
& $(0,52)$ & $(0,09)$ & $(0,12)$ & $(0,00)$ \\
\hline
\end{tabular}

* significativo a10\%; ** significantivo a $5 \%$; *** significativo a $1 \%$. Valor-p entre parênteses

\section{CONCLUSÕES}

Neste texto, procurou-se verificar se existe relação entre a média do retorno sobre o investimento (ROI) e o escore de eficiência médio, medida por meio da metodologia Data Envelopment Analysis (DEA), no período de 2007 a 2009, das empresas analisadas. A amostra foi constituída por 31 empresas de distribuição de energia elétrica brasileiras

Constatou-se que $61 \%$ das empresas tiveram o fator de produtividade total (TFPC) positivo, $45 \%$ o índice de evolução da eficiência técnica (EC) positivo, 100\% o índice da evolução da fronteira tecnológica (TC) positivo, e $30 \%$ o escore do DEA positivo. Determinou-se também o índice do retorno sobre o investimento (ROI) para cada período e a média entre 2007 a 2009, verificando-se que $90 \%$ das empresas apresentaram média positiva neste período.

Calculou-se a correlação do ROI com a variação nos índices TC, EC e TFPC e no escore de eficiência do DEA no período de 2007 a 2009, resultando em uma correlação positiva, porém fraca, entre variação da eficiência com base nos respectivos índices. A correlação negativa entre o ROI e o índice TC pode ser resultado dos custos decorrentes da mudança da fronteira tecnológica, que provocou a redução do lucro operacional. No entanto, o benefício proveniente desta mudança não foi refletido no período em análise. Interessante observar ainda a alta e significativa correlação entre o escore DEA (o DEA tradicional) e o ROI. Isto mostra uma relação importante entre eficiência técnica e retorno financeiro.

\section{AGRADECIMENTOS}

Os autores agradecem à Fundação de Amparo à Pesquisa de Minas Gerais (FAPEMIG), ao Programa Pesquisador Mineiro(FAPEMIG) e ao Conselho Nacional de Desenvolvimento Científico e Tecnológico (CNPq) pelo apoio. 


\section{REFERÊNCIAS}

SILVA, F. A. ; GONÇALVES, C. A. . O processo de formulação e implementação de planejamento estratégico em instituições do setor público. Revista de Administração da UFSM, v. 4, p. 458-476, 2011.

ANEEL: Agencia Nacional de Energia Elétrica. Nota Técnica n. 68/2007 - SER/ANEEL. 2007. Processo no 48500.001208/2006-37. Metodologia e cálculo da taxa de remuneração das concessionárias de distribuição de energia elétrica.

ANEEL. Nota Técnica 265/2010. Metodologia de cálculo dos custos operacionais. Fonte: http://www.aneel.gov.br/aplicacoes/ audiencia/arquivo/2010/040/documento/ nota_tecnica_no_265_custos_operacionais. pdf(acesso em 28/02/2011)

ANEEL. Nota Técnica 267/2010. Metodologia de cálculo do Fator X. Fonte: http:// www.aneel.gov.br/aplicacoes/audiencia/ arquivo/2010/040/documento/nota_tecnica_ no_267_fator_x.pdf(acesso em 28/02/2011)

ÂNGULO-MEZA, L., SOARES DE MELLO, J.C.C.B., GOMES, E.G, FERNANDES, A.J.S. Selecção de variáveis em DEA aplicada a uma análise do mercado de energia eléctrica. Investigação Operacional, v. 27, p. 21-36, 2007.

ARIFF, M., CABANDA, E., SATHYE, M. Privatization and Performance: evidence from telecommunications sector. Journal of the Operational Research Society, v. 60, p. 13151321, 2009.

BANKER, R. D., CHARNES, A., COOPER W. Some models for estimating technical and scale inefficiencies in Data Envelopment Analysis, Management Science, v. 30, p.1078-1092, 1984.

BATH, R., VERMA, B. B., REUBEN, E. A note on data envelopment analysis - DEA. Journal of Health Management, v. 3, n.2, p. 3 9-328, 2001.

CARVALHO, M. B.; GONÇALVES, C. A. ; PARDINI, D. J. . A Felicidade em foco mensurando conceito metafísico para estratégia governamental e recomendações organizacionais. Revista de Administração da UFSM, v. 3, p. 269-287, 2010.

CAVES, D. W., CHRISTENSEN, L. R., DIEWERT, W. E. The Economic Theory of Index Numbers and the Measurement of Input, Output and Productivity, Econometrica, v. 50, n.6, p. 13931414. 1982.

CHARNES, A., COOPER, W. W., RHODES, E. measuring the efficiency of decision making units, European Journal of Operational Research, v. 2, p. 429-444, 1978.

CHERCHYE, L., POST, T., Methodological Advances in DEA: a survey and an application for the Dutch electricity sector. Statistica Neerlandica. V. 57, nr. 4, pp. 410-438. 2003.

DELMAS, M., TOKAT, Y. Deregulation, restructuring and productivity: the U.S. electric utility sector. Annual Conference of the International Society for New Institutional Economics, MIT, 2002.

FÄRE, R., GROSSKOPF, S., NORRIS, M., ZHANG, Z. . Productivity Growth, Technical Progress, and Efficiency Change in Industrialized Countries. The American Economic Review, v. 84, n. 1, p. 66-83, 1994.

FARRELL, James M. The Measurement of Technical Efficiency. Journal of the Royal Statistics Society, SERIES A (GENERAL), PART III, 253-290, 1957.

GOMES, D. T. ; MUNIZ, R. M. ; DIAS, A. T. ; GONÇALVES, C. A. . Apresentação Do Processo De Formação Da Estratégia: Um Estudo De Caso Em Empresa Inovadora. Revista de Administração da UFSM, v. 5, p. 414-437, 2012.

MALMQUIST, S. Index numbers and indifference 
curves. Trabajos de Estatistica, v. 4, n. 1, p. 209-242, 1953.

MARTIC, M., NOVAKOVI, M., BAGGIA, A. Data Envelopment Analysis: basic models and their utilization. Organizacija, Vol. 42, pgs. 37-43, 2009.

RAY, S.C., DESLI, E. Productivity Growth, Technical Progress, and Efficiency Change in Industrialized Countries: Comment. American Economic Review, 87:5, pgs. 1033-1039, 1997.

SANTOS, A. dos, CASA NOVA, S. P. Proposta de um modelo estruturado de análise de demonstrações contábeis. RAE-eletrônica, v. 4, n. 1, Art. 8, jan./jun., 2005.

SARKIS, J., CORDEIRO, J. J. Investigating technical and ecological efficiencies in the electricity generation industry: are there winwin opportunities? Journal of the Operational Research Society, v. 60, p. 1160-1172, 2009.

SENRA, L.F.A.C. Métodos de seleção de variáveis em DEA: estudo de caso no setor elétrico brasileiro. Dissertação (Mestrado em Engenharia de Produção) - Universidade Federal Fluminense, Niterói, Brasil, 2004.

SENRA, L.F.A.C., NANCI, L.C., SOARES DE MELLO, J.C.C.B.; ANGULO MEZA, L. (2007) Estudo sobre métodos de seleção de variáveis em DEA, Pesquisa Operacional, Vol 27, No 2, pp. 191-207.

THRALL, R. M. Measures in DEA with an Applications to the Malmquist Index. Journal of Productivity Analysis 13, pp'. 125-137. 2000.

TORESAN, L. Sustentabilidade e desempenho produtivo na agricultura: uma abordagem multidimensional aplicada a empresas agrícolas. Tese de Doutorado - PPGEP - UFSC, 1998.

WANG, J. H., NGAN, H. W., ENGRIWAN, W., LO, K. L. Performance based regulation of the electricity supply industry in Hong Kong: an empirical efficiency analysis approach. Energy Policy, v. 35, p. 609-615, 2007.

ZHANG, Y., BARTELS, R., The Effect of Sample Size on the Mean Efficiency in DEA with an Application to Electricity Distribution in Australia, Sweden and New Zealand. Journal of Productivity Analysis, 9, 187-204. 1998.

ZHOU, P., ANG, B.W., POH, K.L. A survey of data envelopment analysis in energy and environmental studies. European Journal of Operational Research 189, 2008, pgs. 1-18.

ZHU, J. Quantitative models for performance evaluation and benchmarking. Massachusetts, EUA: Kluwer Academic Publishers, 2003.

ZHU, J. Multi-factor performance measure model with an application to Fortune 500 companies, European Journal of Operational Research, v. 123, p.105-124, 2000. 